\title{
Modelagem de distribuição de espécies arbóreas por classe diamétrica no sudoeste da Amazônia
}

\author{
Modeling the distribution of trees species by diameter class in \\ southwestern Amazonia
}

\author{
Symone Maria de Melo Figueiredo ${ }^{1 *}$ e Evandro Orfanó Figueiredo ${ }^{2}$
}

\begin{abstract}
Resumo
O objetivo do estudo foi analisar a predição da distribuição de espécies florestais madeireiras, em escala local, utilizando dados de ocorrência agrupados por classe diamétrica. Para estimar a distribuição foi utilizado o método de máxima entropia (Maxent) e as ocorrências são de inventário florestal de planos de manejo. As variáveis preditoras dos modelos foram:altitude, declividade, distância vertical à drenagem mais próxima (HAND), índice de vegetação por diferença normalizada (NDVI) e densidade de pontos das espécies florestais. Foram selecionadas seis variáveis preditoras, por espécie, pelo método de todas as regressões possíveis. Os modelos tiveram em média bom desempenho ( $A \cup C=0,7$; taxa de omissão $=8,8 \%$ ), demonstrando a viabilidade de se predizer a distribuição de espécies por classe diamétrica. As variáveis mais importantes foram altitude, NDVI e densidade. De acordo com os modelos, as árvores de Astonium lecointei, Clarisia racemosa e Ceiba pentandra com diâmetro a altura do peito $(\mathrm{DAP}) \geq 100 \mathrm{~cm}$ têm maior probabilidade de ocorrer em ambientes localizados em altitudes mais elevadas do terreno. Esse procedimento de modelagem é eficiente para ampliar o conhecimento sobre as preferências de habitat e a distribuição geográfica de espécies na paisagem em função do DAP.
\end{abstract}

Palavras-chave: Modelos de distribuição de espécies, árvores madeireiras, DAP, Maxent, Brasil.

\begin{abstract}
The aim of the study was to analyze the prediction of the distribution of forest tree species, on a local scale, using occurrence data grouped by diameter class. To estimate the distribution of species the maximum entropy method (Maxent) and the occurrence data are forest management plan was used. The predictor variables were: elevation, slope, height above the nearest drainage (HAND), and normalized difference vegetation index (NDVI) and dot density of forest species. Six predictor variables were selected by species by method of all possible regressions. The models, by species and diameter class, had an average good performance $(A \cup C=0.7$; omission rate $=8.8 \%)$, demonstrating the viability of predicting the distribution of species by diameter class. The most important predictor variables were altitude, NDVI, densities Amburana acreana and Clarisia racemosa. However, further studies are needed to clarify whether there is an interaction between forest species or share the same habitat. According to the models, trees of the species Astonium lecointei, Clarisia racemosa and Ceiba pentandra with diameter at breast height $(\mathrm{DBH}) \geq 100 \mathrm{~cm}$ are more likely to occur in localized higher elevations. This modeling procedure is efficient to increase knowledge about habitat preferences and geographical distribution of species in the landscape by DBH.
\end{abstract}

Keywords: Species distribution models, timber trees, DBH, Brazil.

\section{INTRODUÇÃO}

A modelagem de distribuição de espécies (MDE), em geral, relacionam a distribuição dos pontos de ocorrência da espécie em locais conhecidos com um conjunto multivariado de informações sobre o ambiente desses locais, por meio de ajustes de funções para predizer em que regiões no espaço geográfico é provável sua ocorrência (PHILLIPS et al., 2006).

1. UFAC - Universidade Federal do Acre. Rio Branco, AC, Brasil.

2. Centro de Pesquisa Agroflorestal do Acre, Embrapa - Empresa Brasileira de Pesquisa Agrepecuária. Rio Branco, AC, Brasil.

*Autor Correspondente: symone_ac@yahoo.com.br

Sci. For., Piracicaba, v. 47, n. 124, p. 644-654, dez. 2019

DOI: doi.org/10.18671/scifor.v47n124.06 
Nos últimos anos, têm aumentado o uso e a aplicação da MDE, auxiliando na compreensão dos requerimentos ecológicos das espécies (MILLAR; BLOUIN-DEMERS, 2012) e na tomada de decisão em conservação da biodiversidade (MOTA-VARGAS; ROJAS-SOUTO, 2012; SHCHEGLOVITOVA; ANDERSON, 2013).

A presença e abundância de espécie determinada pela MDE são influenciadas por diferentes variáveis ambientais em função da escala utilizada (MOKANY; ROXBURGH, 2010), capacidade de dispersão das espécies e interações bióticas (GUISAN; THUILLER, 2005; SOBERÓN, 2007).

Na maioria dos casos, os modelos de distribuição de espécies abrangem regiões de grande extensão territorial utilizando variáveis ambientais com resolução mais grosseira, como por exemplo os dados climáticos do WorldClim - Global Climate Data, que tem resolução espacial de $1 \mathrm{~km}$. Em pequenas áreas de estudo $\left(>100 \mathrm{~km}^{2}\right.$ ) são raros os exemplos usados para produzir predições, devido à escassa disponibilidade de variáveis ambientais compatíveis com resoluções finas (pixels >100 m) e à ausência de informações sobre as espécies nessa escala (KHATCHIKIAN et al., 2011).

De acordo com Mokany e Roxburgh (2010) a distribuição das espécies em escala mais grosseira (paisagem) é determinada por fatores climáticos, geológicos e histórico de perturbação, enquanto que em escala mais fina (habitat), por atributos ambientais mais localizados, como fatores topográficos e distúrbios locais. Em escala finíssima ou local (micro-habitat) é mais provável a influência de variação ambiental e interações bióticas (SOBERÓN, 2007).

Nas regiões tropicais, devido à escala mais grosseira das variáveis ambientais e à escassez de dados de ocorrências, os estudos que visam a gestão e o manejo de espécies numa escala local são poucos (CAYUELA et al., 2009; GUISAN; THUILLER, 2005).

Outra limitação é que geralmente são utilizadas apenas variáveis ambientais na construção dos modelos, porém além de condições ambientais adequadas as espécies têm a sua distribuição determinada por outros fatores, como interações bióticas e barreiras geográficas que impedem a dispersão. As barreiras geográficas podem ser tratadas na definição da área de estudo (em vez de limites políticos), mas a inclusão da interação biótica é um desafio na pesquisa em MDE porque geralmente não se tem informação de como uma determinada espécie influencia a existência de outra (GUISAN; THUILLER, 2005).

Para modelar a distribuição de uma espécie em resolução mais fina é recomendado o uso de dados de ocorrência precisos e grandes tamanhos de amostra, proporcionando melhores predições para os organismos sésseis (GUISAN; THUILLER, 2005). Devido à escassez de registros de ocorrência de espécies disponíveis nas coleções de herbários e museus, as informações de inventários florestais pode ser uma alternativa para estudos dessa natureza.

Os inventários florestais são reconhecidos como um recurso valioso para descrever a variação espacial da biomassa, da diversidade, da composição da comunidade e do funcionamento da floresta tropical da América do Sul (TER STEEGE et al., 2006).

No leste do Acre, dados de espécies florestais podem ser obtidos de inventários florestais de planos de manejo cujo planejamento foi realizado segundo os procedimentos do Modelo Digital de Exploração Florestal - Modeflora ${ }^{\circledR}$ (FIGUEIREDO et al., 2007). Os inventários florestais são levantamentos detalhados que contêm, dentre outras, informações sobre a localização e abundância de diversas espécies florestais de interesse madeireiro, dados de altura comercial, diâmetro à altura do peito (DAP), área basal e volume de cada um dos indivíduos inventariados a partir de um determinado diâmetro mínimo.

No manejo florestal, o diâmetro obtido da árvore é uma importante medida do estoque em crescimento, pois afeta o cálculo do volume, área basal, peso e sortimento e possibilita conhecer a distribuição diamétrica da floresta, fornecendo base para identificar a intensidade da regeneração natural em nível de espécie (SCOLFORO; THIERSCH, 2004; SCOLFORO, 2006).

Na maioria das florestas nativas é característica a distribuição diamétrica decrescente ou em forma de "J" invertido (MACHADO et al., 2010; SCOLFORO, 2006), ou seja, a frequência de indivíduos vai diminuindo à medida que aumenta a classe de diâmetro. Diante disso e frente as possibilidades do uso e aplicação da MDE, pergunta-se: existe relação entre o local de ocorrência de espécies em função da classe de tamanho de diâmetro? Em escala fina, além de variáveis ambientais como preditoras dos modelos de distribuição, pode ser também incluída a abundância de outras espécies?

Para responder a essas perguntas, o objetivo do estudo foi analisar a relação entre as classes de tamanho de diâmetro para um grupo de espécies florestais e a distribuição no espaço, em escala local, 
utilizando dados de ocorrência obtidos de inventários florestais de planos de manejo na predição de modelos de distribuição de espécies, incluindo como variáveis preditoras um grupo de variáveis ambientais e biológicas.

\section{MATERIAL E MÉTODOS}

\section{Área de estudo e dados das espécies florestais}

A modelagem de distribuição de espécies florestais de interesse madeireiro foi realizada no leste do estado do Acre, em áreas de Planos de Manejo Florestal Sustentável (PMFS) licenciados pelo Instituto de Meio Ambiente do Acre (Imac), totalizando 34.852 ha. As informações sobre a localização e a extensão dos PMFS estão descritas na Tabela 1.

Tabela 1. Planos de Manejo Florestal Sustentável (PMFS) licenciados pelo Instituto de Meio Ambiente do Acre (Imac) no leste do Acre, Brasil.

Table 1. Sustainable Forest Management Plans (PMFS) licensed by Instituto de Meio Ambiente do Acre (Imac) in eastern Acre, Brazil

\begin{tabular}{cccc}
\hline $\mathbf{N}^{\mathbf{0}}$ & Propriedade & Município & PMFS (ha) \\
\hline 1 & Fazenda Alegria & Manoel Urbano & 282,59 \\
2 & Fazenda Alfenas & Rio Branco & 638,18 \\
3 & Fazenda Belo Horizonte & Rio Branco & $4.711,71$ \\
4 & Fazenda Bom Destino & Rio Branco & $1.598,00$ \\
5 & Fazenda Cajueiro & Bujari & 598,83 \\
6 & Fazenda Canary & Bujari & $7.500,00$ \\
7 & Fazenda Capixaba & Capixaba & 746,44 \\
8 & Fazenda Conquista & Manoel Urbano & $3.204,43$ \\
9 & Fazenda Cutiara & Bujari & 360,11 \\
10 & Fazenda Eliane & Bujari e Rio Branco & $1.208,48$ \\
11 & Fazenda Escorpião & Bujari & 263,16 \\
12 & Fazenda Forquilha & Sena Madureira & $3.873,30$ \\
13 & Fazenda Iquiri & Senador Guiomard & $2.004,46$ \\
14 & Fazenda Santo Antonio & Porto Acre & $2.419,01$ \\
15 & Fazenda Uberaba & Sena Madureira & $5.443,63$ \\
Total & & & $34.852,33$ \\
\hline
\end{tabular}

A MDE foi realizada para um grupo de oito espécies florestais (Tabela 2) com ocorrências nos PMFS (Tabela 1). Para a confirmação do nome científico das espécies selecionadas para a modelagem, foram realizadas nas áreas de PMFS, coletas de material botânico para herborização, com o seguinte número de registro: UFACPZ 20676, UFACPZ 20677, UFACPZ 20678, UFACPZ 20679, UFACPZ 20680, UFACPZ 20681, NY01488096, NY01488095, NY01488099, NY01488098, NY01488097, NY01488100, RB00825038, RB 589540, RB 589539, RB 592020, RB 589544, RB 592024, RB 592028, RB 589543, RB 592020, RB 589544, RB 592024, RB 592028, RB 589543. As exsicatas foram depositadas no herbário do Parque Zoobotânico da Universidade Federal do Acre (UFACPZ), utilizando para a identificação o sistema de classificação Cronquist (1981). Além disso, para algumas espécies, foram enviadas exsicatas a especialistas do The New York Botanical Garden e do Jardim Botânico do Rio de Janeiro.

O banco de dados dos inventários florestais dos PMFS foram disponibilizados pela Embrapa Acre e pelo Imac. Os dados utilizados na modelagem foram: a) os registros de ocorrência das árvores individuais, georreferenciadas com Sistema de Posiciamento Global (GPS) modelo Garmin 76csx, e b) diâmetro à altura do peito (DAP).

Para cada espécie, os registros de ocorrência foram compilados e divididos de acordo com o tamanho do diâmetro à altura do peito (DAP) em quatro classes com intervalo de $20 \mathrm{~cm}$ (Tabela 3). Para determinar a amplitude das classes diamétricas foi adotado como critério um número mínimo de dados de ocorrência em cada classe, que permitisse a construção dos modelos de distribuição de espécies. 
Tabela 2. Relação das espécies florestais e variáveis preditoras selecionadas para a modelagem de distribuição potencial no leste do Acre, Brasil.

Table 2. List of forest species and predictor variables selected for potential distribution modeling in eastern Acre, Brazil.

\begin{tabular}{|c|c|c|c|c|}
\hline Id & Nome científico & Família & Nome popular & Variáveis preditoras \\
\hline 1 & Amburana acreana (Ducke) A.C.Sm. & Fabaceae & $\begin{array}{c}\text { Cerejeira, } \\
\text { cumaru-de-cheiro. }\end{array}$ & $\begin{array}{c}\text { HAND, NDVI, } D_{\text {Aroeira }}, D_{\text {Assacu }} \\
D_{\text {Garapeira }}, D_{\text {Guariúba }}\end{array}$ \\
\hline 2 & Apuleia leiocarpa (Vogel) J.F.Macbr. & Fabaceae & $\begin{array}{l}\text { Cumaru-cetim, } \\
\text { garapeira }\end{array}$ & $\begin{array}{l}\text { Altitude, declividade, NDVI, } \\
\qquad D_{\text {Aroeira }}, D_{\text {Caucho }}, D_{\text {Pereiro }}\end{array}$ \\
\hline 3 & Aspidosperma macrocarpon Mart. & Apocynaceae & Pereiro & $\begin{array}{l}\text { Altitude, HAND, } D_{\text {Caucho' }} \\
D_{\text {Cerejeira, }}, D_{\text {Guariúba }}\end{array}$ \\
\hline 4 & Astronium lecointei Ducke. & Anacardiaceae & $\begin{array}{l}\text { Maracatiara, } \\
\text { aroeira. }\end{array}$ & $\begin{array}{l}\text { Altitude, declividade, HAND, } \\
\text { NDVI, } D_{\text {Assacu }}, D_{\text {Samaúma }}\end{array}$ \\
\hline 5 & Castilla ulei Warb. & Moraceae & Caucho & $\begin{array}{c}\text { NDVI, } D_{\text {Assacu }}, D_{\text {Cerejeira, }}, D_{\text {Guariúba }}, \\
D_{\text {Pereiro }}, D_{\text {Samaúma }}\end{array}$ \\
\hline 6 & Ceiba pentandra (L.) Gaertn. & Malvaceae & Samaúma & $\begin{array}{c}\text { HAND, } D_{\text {Aroeira }}, D_{\text {Assacu }}, D_{\text {Cerejeira }}, \\
D_{\text {Garapeira }}, D_{\text {Guariúba }}\end{array}$ \\
\hline 7 & Clarisia racemosa Ruiz \&Pav & Moraceae & Guariúba & $\begin{array}{c}\text { Altitude, } D_{\text {Aroeira }}, D_{\text {Assacu' }}, D_{\text {Caucho' }}, \\
D_{\text {Pereiro }}, D_{\text {Samaúma }}\end{array}$ \\
\hline 8 & Hura crepitans L. & Euphorbiaceae & Assacu ou açacu & $\begin{array}{c}\text { Altitude, HAND, } D_{\text {Aroeira }}, D_{\text {Caucho }}, \\
D_{\text {Garapeira }}, D_{\text {Samaúma }}\end{array}$ \\
\hline
\end{tabular}

Tabela 3. Número total de indivíduos por classe diamétrica das espécies florestais presentes nos planos de manejo florestal no leste do Acre, Brasil: a) Astronium lecointei; b) Hura crepitans; c) Castilla ulei; d) Amburana acreana; e) Apuleia leiocarpa; f) Clarisia racemosa; g) Aspidosperma macrocarpon; h) Ceiba pentandra.

Table 3. Total number of individuals per diameter class of the forest species present in the forest management plans in eastern Acre, Brazil: a) Astronium lecointei; b) Hura crepitans; c) Castilla ulei; d) Amburana acreana; e) Apuleia leiocarpa; f) Clarisia racemosa; g) Aspidosperma macrocarpon; h) Ceiba pentandra.

\begin{tabular}{cccccccccc}
\hline $\begin{array}{c}\text { Classe } \\
\text { de DAP }\end{array}$ & $\begin{array}{c}\text { Intervalo } \\
(\mathbf{c m})\end{array}$ & $\mathbf{a}$ & $\mathbf{b}$ & $\mathbf{c}$ & $\mathbf{d}$ & $\mathbf{e}$ & $\mathbf{f}$ & $\mathbf{g}$ & $\mathbf{h}$ \\
\hline 1 & $40+60$ & 2.299 & 501 & 8.149 & 1.012 & 2.092 & 4.923 & 250 & 324 \\
2 & $60+80$ & 1.169 & 675 & 3.918 & 863 & 2.844 & 2.451 & 148 & 539 \\
3 & $80+100$ & 210 & 588 & 538 & 312 & 1.846 & 572 & 78 & 541 \\
4 & $\geq 100$ & 37 & 1.230 & 32 & 47 & 636 & 93 & 19 & 1.143 \\
Total & & $\mathbf{3 . 7 1 5}$ & $\mathbf{2 . 9 9 4}$ & $\mathbf{1 2 . 6 3 7}$ & $\mathbf{2 . 2 3 4}$ & $\mathbf{7 . 4 1 8}$ & $\mathbf{8 . 0 3 9}$ & $\mathbf{4 9 5}$ & $\mathbf{2 . 5 4 7}$ \\
\hline
\end{tabular}

\section{Variáveis preditoras}

Devido à escala fina definida para o estudo, ou seja, variáveis ambientais com resolução espacial de $90 \mathrm{~m}$ e área de estudo com aproximadamente $35 \mathrm{mil} \mathrm{ha}$, foram usadas variáveis topográficas e de índice de vegetação para caracterizar o ambiente em curtas distâncias. Para expressar a interação biológica entre as espécies florestais foram utilizados os dados de densidade de indivíduos. Assim, as variáveis preditoras foram organizadas em dois grupos: ambientais e biológicas.

As variáveis ambientais topográficas, derivadas de dados do Shuttle Radar Topographic Mission (SRTM) com resolução de $90 \mathrm{~m}$, foram: altitude, declividade e distância vertical à drenagem mais próxima (Height Above the Nearest Drainage - HAND). A HAND foi processada de acordo com a metodologia de Rennó et al. (2008). O índice de vegetação por diferença normalizada (NDVI) foi obtido a partir de um mosaico de imagens do Landsat TM (Thematic Mapper), reamostrado para resolução de 90 m, compatível com as demais variáveis. As imagens são do período seco do ano de 2011, por ser uma data anterior à exploração florestal nas áreas manejadas.

As variáveis biológicas representam a superfície de densidade de pontos de cada uma das espécies florestais de interesse madeireiro, que foram construídas usando o banco de dados das ocorrências registradas nos 15 inventários florestais dos planos de manejo. O valor da densidade de pontos de cada pixel corresponde à média da contagem do número de ocorrência dos pixels vizinhos, considerando uma janela de 5 x 5 pixel. Dessa forma, quanto maior o número de indivíduos por unidade de área, 
maior o valor de densidade. O processamento das variáveis ambientais e biológicas (densidades) foi realizado em softwares de sistema de informação geográfica e de processamento de imagem digital.

Para a seleção das variáveis explicativas dos modelos de distribuição das espécies por classe diamétrica foi utilizado o método de todas as regressões possíveis (RYAN, 2011), que pondera a inclusão de 2 a 6 parâmetros $\left(\beta_{\text {is }}\right)$, considerando os critérios: sem multicolinariedade, com distribuição normal dos resíduos, independentes e homocedásticos, para isso, são realizadas as seguintes estatísticas: matriz de multicolinariedade, para indicação das variáveis explicativas a serem empregadas nos modelos; distribuição do erro padronizado e o teste de aderência Kolmogorov Smirnov, para normalidade; teste de Durbin-Watson, para independência dos resíduos e o F-máximo de Hartley, para avaliar a homocedasticidade (STATGRAPHICS, 2006).

Nesse procedimento, a variável dependente foi o diâmetro à altura do peito (DAP) e as variáveis explicativas foram as quatro preditoras ambientais e as sete densidades de pontos das outras espécies também presentes na área. As variáveis selecionadas foram aquelas cuja regressão apresentou o menor quadrado médio do erro (QMR) e o maior coeficiente de determinação ajustado ( $\mathrm{R}_{\text {ajust }}^{2}$ ). Esse procedimento foi repetido para cada uma das oito espécies e as variáveis selecionadas estão relacionadas na Tabela 1.

\section{Modelo utilizado}

Na modelagem da distribuição de espécies foi usado o algoritmo de máxima entropia (Maxent), um método para realizar predição ou inferências baseado apenas em dados de presença (PHILIPS et al., 2006), utilizando o software Maximum Entropy Species Distribution Modelling v. 3.3.3k (<www.cs.princeton.edu/_schapire/maxent/>).

O Maxent vem sendo usado a partir de 2004 na modelagem de distribuição de espécies, estimando a probabilidade de adequação do habitat onde é possível a ocorrência da espécie, encontrando a distribuição de probabilidade de máxima entropia (mais próxima do uniforme), que é sujeita a um conjunto de restrições, no qual os valores esperados em cada feição (variáveis ambientais) devem corresponder à suas médias observadas nas amostras (PHILLIPS et al., 2006; ELITH et al., 2011).

Para cada espécie selecionada foi gerado um conjunto de cinco modelos Maxent: um modelo por classe de DAP (ocorrências divididas em quatro classes) e um modelo geral, incluindo o conjunto de ocorrências sem a divisão por classe diamétrica. Os números de ocorrências disponíveis para a modelagem estão citados na Tabela 2.

Na construção de cada modelo foram feitas 10 partições aleatórias dos dados de ocorrência, usando a técnica de reamostragem subsample, gerando 10 subamostras do conjunto de dados, sem reposição. Em cada subamostra os dados foram divididos aleatoriamente, 70\% para amostras de treino e 30\% para amostras de teste externo.

Para estimar quais são as variáveis mais importantes no modelo, foi usado o teste de Jackknife, tanto para reduzir o viés de variáveis ambientais correlacionadas quanto para diagnosticar quais variáveis ambientais representam o preditor mais importante para gerar o MDE (PRATES-CLARK et al., 2008)

Para a avaliação do desempenho dos modelos foram analisados os resultados da área sob a curva (Area Under the Curve - AUC) e das taxas de omissão dos modelos, considerada importante por Lobo et al. (2008), como uma medida auxiliar à AUC.

A AUC é interpretada como a probabilidade do modelo classificar um local de presença escolhido aleatoriamente em relação a um local qualquer da paisagem (MEROW et al., 2013). Um modelo aleatório tem uma AUC de 0,5 e quanto mais próximo de 1,0 melhor o seu desempenho (PHILLIPS et al., 2006).

Para o cálculo da taxa de omissão do modelo foi usado o limiar 10 Percentile Training Presence, o qual assume que $10 \%$ dos registros de treinamento do algoritmo com os menores valores de probabilidade serão descartados. Esse limiar foi escolhido por ser mais rigoroso, visto que a avaliação ocorreu dentro das áreas dos planos de manejo.

Foi aplicado o teste t para avaliar a significância $(\alpha=0,05)$ da variação em torno da média do tamanho das áreas de predição dos modelos por classe de diâmetro e ainda foram computadas as áreas de consenso entre os modelos para avaliar se a área de projeção geográfica difere ou não em função do tamanho de DAP. 


\section{RESULTADOS E DISCUSSÃO}

\section{Distribuição diamétrica}

O resultado obtido da distribuição de frequência por classe diamétrica (Figura 1) mostrou uma concentração de indivíduos nas menores classes de DAP em forma de J invertido, indicando um alto grau de regeneração natural para A. acreana, A. leiocarpa, A. macrocarpon, A. lecointei, C. ulei e C. racemosa.

H. crepitans

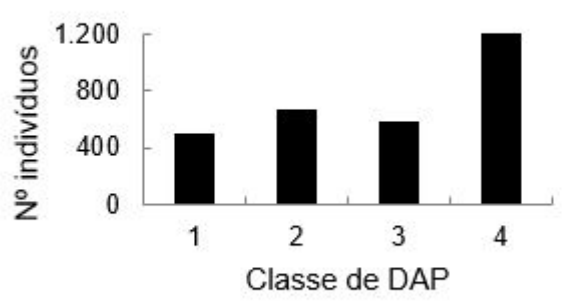

C. ulei

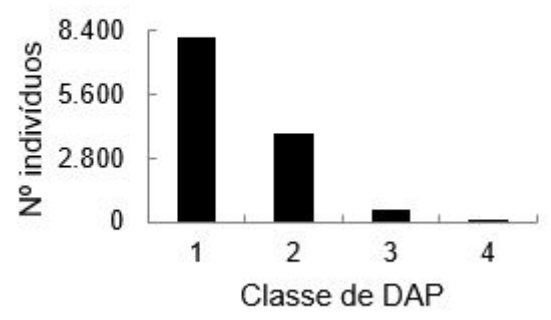

A. leiocarpa

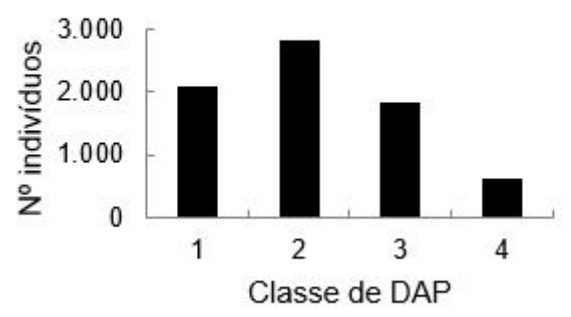

A. macrocarpon

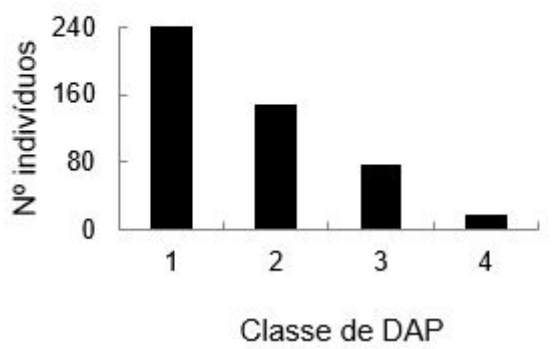

A. lecointei

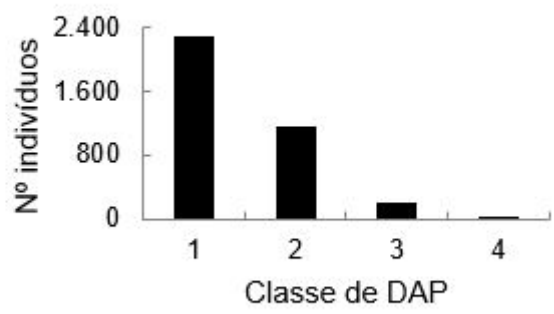

A. acreana

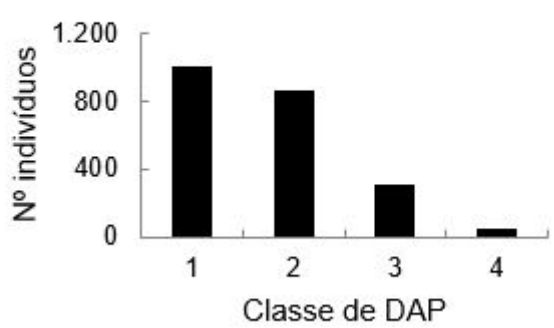

C. racemosa

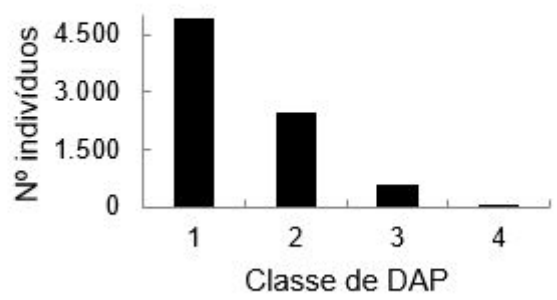

C. pentandra

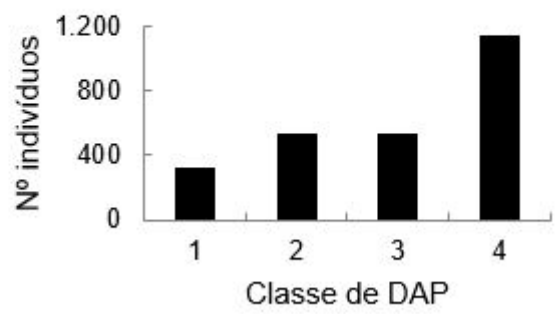

Figura 1. Distribuição diamétrica das espécies florestais utilizadas na modelagem localizadas em planos de manejo florestal no leste do Acre, Brasil. $1=$ classe $1(40 \mathrm{~cm}+60 \mathrm{~cm}) ; 2=$ classe $2(60 \mathrm{~cm}+80 \mathrm{~cm}) ; 3=$ classe 3 $(80 \mathrm{~cm}+100 \mathrm{~cm}) ; 4=$ classe $4(\geq 100 \mathrm{~cm})$.

Figure 1. Diametric distribution of forest species used in modeling located in forest management plans in eastern Acre, Brazil. $1=$ class $1(40 \mathrm{~cm}+60 \mathrm{~cm}) ; 2=\operatorname{class} 2(60 \mathrm{~cm}+80 \mathrm{~cm}) ; 3=\operatorname{class} 3(80 \mathrm{~cm}+100 \mathrm{~cm})$; $4=$ class $4(\geq 100 \mathrm{~cm})$. 
A distribuição dos diâmetros de C. pentandra e H. crepitans apresentou uma curva com forte assimetria à direita, com a maioria dos indivíduos na classe de DAP $\geq 100 \mathrm{~cm}$. Machado et al. (2010) e Scolforo (2006) afirmam que a distribuição decrescente ou em forma de J invertido é característica da maioria das florestas nativas, porém distribuições unimodais podem ser encontradas quando se consideram populações de uma única espécie.

\section{Métricas de avaliação dos modelos e importância das variáveis preditoras}

O desempenho dos modelos de distribuição de espécies foi satisfatório, com valores de AUC $\geq 0,7$ e taxas de omissão $\leq 10 \%$, em $67,5 \%$ dos modelos testados (Tabela 4). Considerando que os resultados da modelagem obtidos em outros estudos, com o uso de dados de ocorrência de inventários florestais na Amazônia brasileira e peruana (FIGUEIREDO et al., 2015; 2016; PÉREZ CHAVES et al., 2018), obtiveram valores de AUC que oscilaram entre 0,6 a 0,9, como nesse trabalho, mostra que é possível realizar a modelagem com o uso de ocorrências agrupados por classe de DAP.

Tabela 4. Valores de AUC e taxa de omissão (\%) de teste externo dos modelos de distribuição de espécie por classe diamétrica e geral: a) Astronium lecointei; b) Hura crepitans; c) Castilla ulei; d) Amburana acreana; e) Apuleia leiocarpa; f) Clarisia racemosa; g) Aspidosperma macrocarpon; h) Ceiba pentandra.

Table 4. AUC values and omission rate (\%) of external test of the species distribution models by diameter and general class: a) Astronium lecointei; b) Hura crepitans; c) Castilla ulei; d) Amburana acreana; e) Apuleia leiocarpa; f) Clarisia racemosa; g) Aspidosperma macrocarpon; h) Ceiba pentandra.

\begin{tabular}{|c|c|c|c|c|c|c|c|c|}
\hline DAP (cm) & $a$ & b & C & d & e & $f$ & g & h \\
\hline \multirow{2}{*}{$40+60$} & 0,6 & 0,7 & 0,7 & 0,7 & 0,7 & 0,6 & 0,9 & 0,7 \\
\hline & $9 \%$ & $11 \%$ & $10 \%$ & $9 \%$ & $8 \%$ & $4 \%$ & $5 \%$ & $12 \%$ \\
\hline \multirow[t]{2}{*}{$60+80$} & 0,6 & 0,7 & 0,7 & 0,6 & 0,7 & 0,7 & 0,8 & 0,6 \\
\hline & $12 \%$ & $15 \%$ & $11 \%$ & $11 \%$ & $10 \%$ & $10 \%$ & $10 \%$ & $13 \%$ \\
\hline \multirow[t]{2}{*}{$80+100$} & 0,7 & 0,7 & 0,7 & 0,7 & 0,6 & 0,7 & 0,9 & 0,7 \\
\hline & $10 \%$ & $14 \%$ & $6 \%$ & $9 \%$ & $11 \%$ & $6 \%$ & $9 \%$ & $7 \%$ \\
\hline \multirow[t]{2}{*}{$\geq 100$} & 0,9 & 0,7 & 0,7 & 0,6 & 0,6 & 0,8 & 0,9 & 0,6 \\
\hline & $0 \%$ & $7 \%$ & $0 \%$ & $14 \%$ & $15 \%$ & $7 \%$ & $0 \%$ & $9 \%$ \\
\hline \multirow[t]{2}{*}{ Geral } & 0,6 & 0,7 & 0,7 & 0,6 & 0,6 & 0,6 & 0,8 & 0,6 \\
\hline & $10 \%$ & $8 \%$ & $9 \%$ & $11 \%$ & $11 \%$ & $5 \%$ & $8 \%$ & $10 \%$ \\
\hline
\end{tabular}

Os resultados do teste Jacknife permitem inferir que entre as variáveis ambientais utilizadas, a altitude e o NDVI foram aquelas com maior percentual de importância entre os modelos avaliados (Figura 2), assim como relatado em outros estudos, que o uso dessas variáveis, derivadas de sensoriamento remoto, também foram as mais importantes na MDE (FIGUEIREDO et al., 2015; PÉREZ CHAVES et al., 2018).
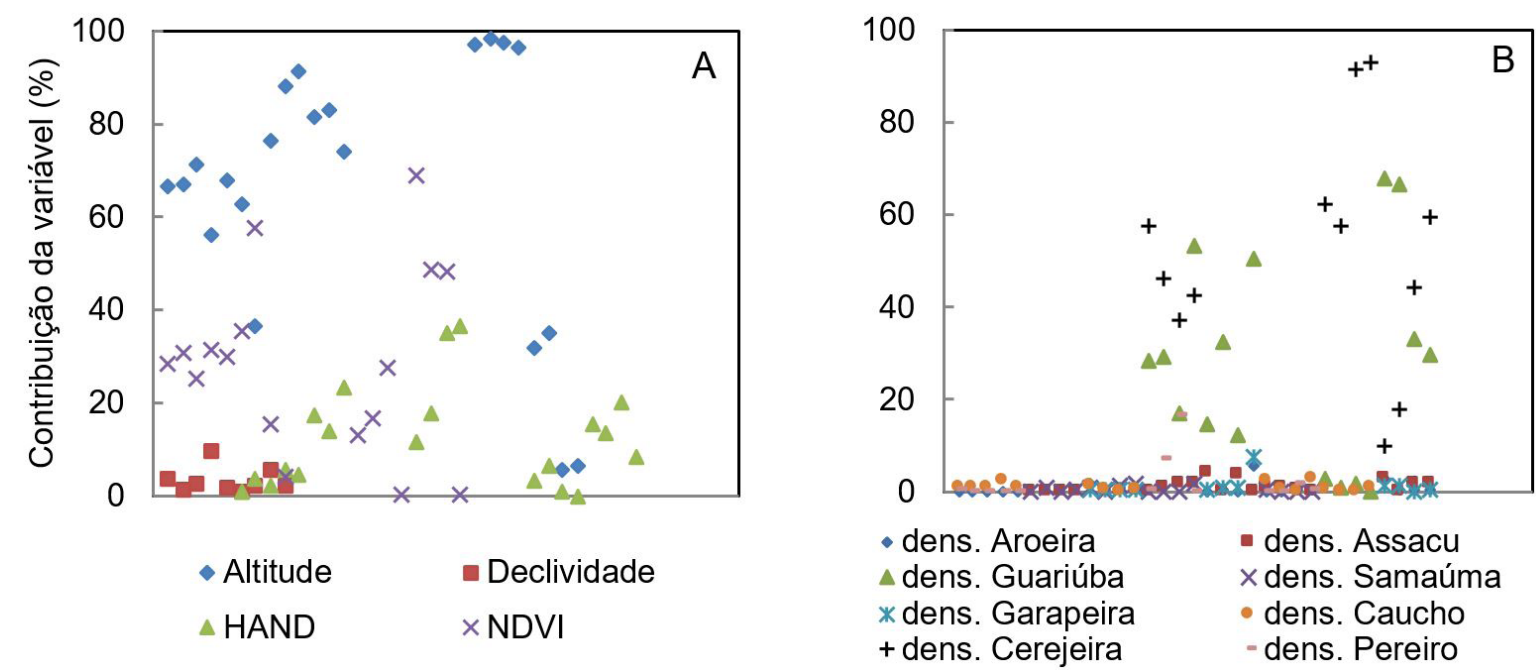

Figura 2. Contribuição percentual das variáveis ambientais (A) e biológicas (B) nos modelos Maxent no leste do Acre, Brasil. dens. $=$ densidade.

Figure 2. Percentage contribution of environmental $(A)$ and biological $(B)$ variables in Maxent models in eastern Acre, Brazil. dens. = density. 
No entanto, verificamos que a combinação entre variáveis ambientais e variáveis biológicas tem informações importantes na predição da distribuição de espécies. Em 37,5\% dos modelos avaliados, as informações mais importantes na distribuição predita foram associadas a uma variável biológica, mostrando que a densidade de ocorrência de outras espécies pode ser usada na predição de modelos em pequena escala.

Na modelagem de C. ulei, A. acreana e C. pentandra, a variável associada à densidade de C. racemosa foi considerada como responsável por uma contribuição percentual entre $12,4 \%$ e $68 \%$ nos modelos por classe diamétrica. C. racemosa é uma espécie generalista (FIGUEIREDO et al., 2015) com distribuição ampla e capacidade de desenvolvimento em variados habitats, exceto em florestas alagadas, onde ocorre raramente (CARDONA-PEÑA et al., 2005), assim, podem ocorrer associações com outras espécies, compartilhando características ecológicas e uso de habitats semelhantes.

Nos modelos para três espécies florestais madeireiras, a densidade de A. acreana foi a variável que parece ter a informação mais útil que não está presente nas outras variáveis. Esse resultado se repetiu nos modelos de distribuição de C. ulei e A. macrocarpon, independentemente da classe diamétrica, e também na predição da distribuição de $C$. pentandra com DAP $\geq 80 \mathrm{~cm}$.

Isso pode refletir que a distribuição de C. ulei, A. macrocarpon e os indivíduos de C. pendantra com $\mathrm{DAP} \geq 80 \mathrm{~cm}$, ocorrem em áreas mais altas do terreno, pois nessas condições é maior a densidade de A. acreana. A confirmação de que as árvores de maior tamanho de C. pentandra estão localizadas no topo é sugerida pela análise dos resultados de modelos construídos com as ocorrências com os maiores DAPs, pois a importância da variável distância vertical à drenagem mais próxima (HAND) diminuiu em relação às classes de menores diâmetros.

As interações bióticas afetam a distribuição (SOBERÓN; PETERSON, 2005) e devem ser consideradas nos modelos. Os resultados mostraram que a densidade de ocorrência de outras espécies pode ser usada na predição de modelos em pequena escala, seja por uma interação biótica ainda não esclarecida ou mesmo em substituição a uma informação que não está presente nas variáveis ambientais.

Apesar de não ser claro se a utilização da ocorrência de uma espécie na modelagem de outra espécie reflete verdadeiramente uma interação biótica (GUISAN; THUILLER, 2005), esse é um tema novo e pouco explorado em modelagem de distribuição de espécies. Portanto, se faz necessário ampliar os estudos para esclarecer de que forma a existência de uma espécie num determinado local influencia a ocorrência de outra.

\section{Tamanho da área predita por classe de DAP e projeção no espaço geográfico}

A extensão da área predita como possível presença das espécies florestais por classe de diâmetro oscilou entre aproximadamente 14,0 e 18,4 mil hectares, exceto para A. macrocarpon que variou em torno de 8,5 a 10,0 mil hectares (Tabela 5). Para todas as espécies, a área prevista como presença por classe diamétrica não apresentou diferença estatística significativa em relação à média $(p>0,05$, teste t).

Tabela 5. Área predita como possível presença (ha) pelos modelos de distribuição de espécie por classe diamétrica nos planos de manejo florestal no leste do Acre, Brasil.

Table 5. Area predicted as possible presence (ha) by the species distribution models by diameter class in the forest management plans in eastern Acre, Brazil.

\begin{tabular}{lrrrrrr}
\hline \multicolumn{1}{c}{ Classe de DAP } & $\mathbf{4 0}+\mathbf{6 0}$ & $\mathbf{6 0}+\mathbf{8 0}$ & $\mathbf{8 0}+\mathbf{1 0 0}$ & $\mathbf{2 1 0 0}$ & Média & $\begin{array}{c}\text { Desvio } \\
\text { padrão }\end{array}$ \\
\hline Astronium leicointei & $17.613,5$ & $18.390,2$ & $18.812,3$ & $14.611,6$ & $17.356,9$ & $1.896,3$ \\
Hura crepitans & $14.028,4$ & $14.912,9$ & $15.533,4$ & $16.596,9$ & $15.267,9$ & $1.080,0$ \\
Castilla ulei & $14.873,2$ & $16.353,9$ & $15.847,7$ & $18.419,4$ & $16.373,5$ & $1.495,9$ \\
Amburana acreana & $18.413,7$ & $17.551,9$ & $18.571,7$ & $18.613,0$ & $18.287,6$ & 497,9 \\
Apuleia leiocarpa & $16.035,6$ & $16.212,2$ & $17.357,5$ & $16.541,0$ & $16.536,6$ & 586,0 \\
Clarisia racemosa & $18.515,0$ & $18.509,3$ & $18.358,7$ & $16.567,7$ & $17.987,7$ & 949,4 \\
Aspidosperma macrocarpon & $8.593,3$ & $9.902,3$ & $8.616,0$ & $8.544,7$ & $8.914,1$ & 659,5 \\
Ceiba pentandra & $18.007,1$ & $17.299,2$ & $17.462,8$ & $17.990,1$ & $17.689,8$ & 362,9 \\
\hline
\end{tabular}

Em relação à área de projeção geográfica, na sua maioria houve consenso de mais de $70 \%$ entre as áreas preditas por todos os modelos de distribuição potencial de espécies, independente da classe diamétrica. Porém, nos modelos de A. lecointei, C. racemosa e C. pentandra foi verificado maior probabilidade de ocorrência das árvores com DAP $\geq 100 \mathrm{~cm}$ nas cotas mais elevadas do terreno (Figura 3). 


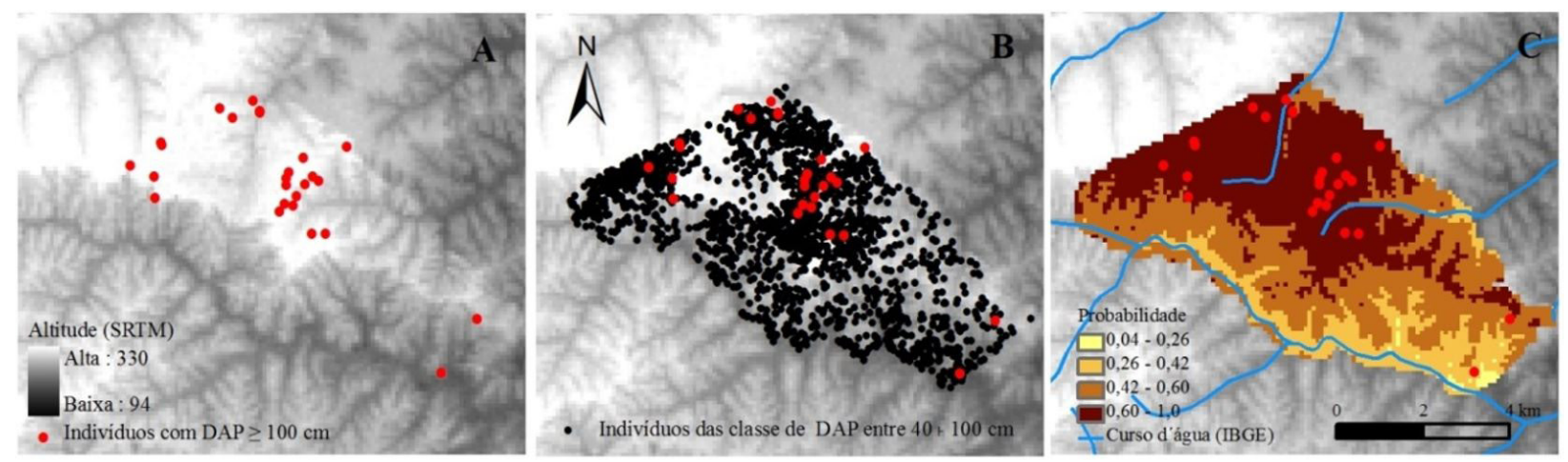

Figura 3. Altitude (A), ocorrência observada (B) e adequabilidade ambiental pelo método Maxent (C) para Clarisia racemosa na Fazenda Uberaba, Acre, Brasil.Os pontos são ocorrências de indivíduos com DAP $\geq 100 \mathrm{~cm}$ (vermelho) e com DAP $<100 \mathrm{~cm}$ (preto).

Figure 3. Elevation (A), observed occurrence (B) and environmental suitability by the Maxent method (C) for Clarisia racemosa at Fazenda Uberaba, Acre, Brazil. Dots are occurrences of individuals with $\mathrm{DBH} \geq 100 \mathrm{~cm}$ (red) and with $\mathrm{DBH}<100 \mathrm{~cm}$ (black).

Na modelagem para as espécies A. lecointei e C. racemosa, os resultados mostraram que há uma tendência de diminuir a concordância entre as áreas preditas pelos modelos à medida que aumenta o DAP. Essa relação mostra que os indivíduos dessas espécies ocupam diferentes locais no espaço geográfico em função da classe de diâmetro, particularmente aqueles com DAP $\geq 100 \mathrm{~cm}$, que pelos registros de ocorrência e os resultados da modelagem, estão mais agregados em locais com maior altitude, onde os modelos mostraram maior probabilidade de possível presença.

Valencia et al. (2004) verificaram que a área basal e densidade de árvores de espécies florestais na Amazônia equatoriana é maior nos habitas das regiões mais elevadas do terreno, diminuindo gradativamente nas áreas inclinadas e nos vales. Na Amazônia central brasileira, Castilho et al. (2006) constataram que a biomassa de árvores tende a aumentar em solos ricos em argila, geralmente localizados nas áreas mais altas do terreno, e que a biomassa é altamente correlacionada com o diâmetro do tronco.

Dessa forma, a distribuição das árvores de maior diâmetro de A. lecointei, C. racemosa e C. pentandra pode ter sido influenciada pela variação química e de textura dos solos, disponibilidade de luz e/ou nas características adaptativas dessas espécies, ao longo de um gradiente topográfico (RESS et al., 2001; TUOMISTO et al., 2003; VALENCIA et al., 2004).

\section{CONCLUSÕES}

A predição da distribuição de espécies florestais madeireiras pode ser feita por classe diamétrica, em pequena escala, com desempenho satisfatório, considerando que a AUC variou de 0,6 a 0,9 com taxas de omissão entre $0 \%$ a $15 \%$.

Não foi verificada uma distinção entre o tamanho das áreas de distribuição potencial em função da classe diamétrica nos modelos de A. acreana, A. leiocarpa, A. macrocarpon, A. lecointei, C. ulei, C. racemosa, C. pentandra e H. crepitans, nos planos de manejo florestal no leste do Acre.

A maior probabilidade de presença de indivíduos de A. lecointei, C. racemosa e C. pentandra com DAP $\geq 100 \mathrm{~cm}$ ocorre em ambientes com as altitudes mais elevadas do terreno.

Apesar dos avanços obtidos nos procedimentos e na metodologia aplicados à modelagem de distribuição de espécie em escala local apresentadas nesse trabalho, novos estudos são importantes e essenciais para esclarecer se dados de densidade de espécies florestais têm informações importantes que podem influenciar a ocorrência de outra.

\section{REFERÊNCIAS BIBLIOGRÁFICAS}

CARDONA-PEÑA, V.; FUENTES, A.; CAYOLA, L. Las moráceas de la región de Madidi, Bolivia. Ecología en Bolivia, La Paz, v. 40, n. 3, p. 212-264, 2005.

CASTILHO, C. V.; MAGNUSSON; W. E.; ARAÚJO, N. O.; LUIZÃO, R. C. C.; LUIZÃO, F. J.; LIMA, A. P.; HIGUCHI, $\mathrm{N}$. Variation in aboveground tree live biomass in a central Amazonian Forest: Effects of soil and topography. Forest ecology and management, Amsterdam, v. 234,p. 85-96. 2006. 
CAYUELA, L.; GOLICHER, D. J.; NEWTON, A. C.; KOLB, M.; ALBURQUERQUE, F. S. DE; ARETS, E. J. M. M.; ALKEMADE, J. R. M.; PÉREZ, A. M. Species distribution modeling in the tropics: problems, potentialities, and the role of biological data for effective species conservation. Tropical Conservation Science, Menlo Park, v. 2, n. 3, p. 319-352, 2009.

CRONQUIST, A. Na integrated system of classification off lowering plants. New York: Columbia University Press, 1981.

ELITH, J.; PHILLIPS; S. J.; HASTIE, T.; DUDÍK, M; CHEE, Y. E.; YATES, C. J. A statistical explanation of MaxEnt for ecologists. Diversity and Distributions, New Jersey, v. 17, n. 1, p. 43-57, 2011.

FIGUEIREDO, S. M. M.; VENTICINQUE, E. M.; FIGUEIREDO, E. O. Spatial scale effects of sampling on the interpolation of species distribution models in the Southwestern Amazon. Revista Árvore, Viçosa, v. 40, n. 4, p. 617-625, 2016.

FIGUEIREDO, S. M. M.; VENTICINQUE, E. M.; FIGUEIREDO, E. O.; FERREIRA, E. J. L. Predição da distribuição de espécies florestais usando variáveis topográficas e de índice de vegetação no leste do Acre, Brasil. Acta Amazonica, Manaus, v. 45, n. 2, p. 167-174, 2015.

FIGUEIREDO, E.O.; BRAZ, E.M.; D'OLIVEIRA, M.V.N. Manejo de Precisão em Florestas Tropicais: Modelo Digital de Exploração Florestal. Rio Branco: Embrapa Acre, 2007, 183 p.

GUISAN, A.; THUILLER, W.Predicting species distribution: offering more than simple habitat models. Ecology letters, Oxford, v. 8, n. 9, p. 993-1009, 2005.

KHATCHIKIAN, C.; SANGERMANO, F.; KENDELL, D.; LIVDAHL, T. Evaluation of species distribution model algorithms for fine scale container breeding mosquito risk prediction. Medical and veterinary entomology, Oxford, v. 25, n. 3, p. 268-275, 2011.

LOBO, J. M.; JIMÉNEZ VALVERDE, A.; REAL, R. AUC: a misleading measure of the performance of predictive distribution models. Global ecology and Biogeography, Oxford, v. 17, n. 2, p. 145-151, 2008.

MACHADO, S. A.; SANTOS, A. A. P.; NASCimENTO, R. G. M.; AUGUSTYNCZIK, A. L. D.; ZAMIN, N. T. Modelagem da distribuição diamétrica de quatro espécies de Lauraceae em um fragmento de Floresta Ombrófila Mista. RECEN-Revista Ciências Exatas e Naturais, Guarapuava, v. 12, n. 1, p. 91-105, 2010.

MEROW, C.; SMITH, M. J.; SILANDER, J. A. A practical guide to MaxEnt for modeling species' distributions: what it does, and why inputs and settings matter. Ecography, Copenhagen, v. 36, n. 10, p. 1058-1069, 2013.

MILLAR, C.S.; BLOUIN-DEMERS, G. Habitat suitability modelling for species at risk is sensitive to algorithm and scale: A case study of Blanding's turtle, Emydoidea blandingii, in Ontario, Canada. Journal for Nature Conservation, Cork, v. 20, n. 1, p. 18-29, 2012.

MOKANY, K; ROXBURGH, S.H. On testing predictions of species relative abundance from maximum entropy optimisation. Oikos, Copenhagen, v. 119, n. 4, p. 583-590, 2010

MOTA-VARGAS, C.; ROJAS-SOTO, O.R. The importance of defining the geographic distribution of species for conservation: The case of the Bearded Wood-Partridge. Journal for Nature Conservation, Cork, v. 20, n. 1, p. $10-17,2012$.

PÉREZ CHAVES, P.; RUOKOLAINEN, K.; TUOMISTO, H. Using remote sensing to model tree species distribution in Peruvian lowland Amazonia. Biotropica, Lawrence, v. 50, n. 5, p. 758-767, 2018.

PHILLIPS, S. J.; ANDERSON, R. P.; SCHAPIRE, R. E. Maximum entropy modeling of species geographic distributions. Ecological modelling, Amsterdam, v. 190, n. 3, p. 231-259, 2006.

PRATES-CLARK, C.C.; SAATCHI, S. S.; AGOSTI, D. Predicting geographical distribution models of high-value timber trees in the Amazon Basin using remotely sensed data. Ecological Modelling, Amsterdan, v. 211, n. 3-4, p. 309-323, 2008.

REES, M.; CONDIT, R.; CRAWLEY, M.; PACALA, S.; TILMAN, D. Long-term studies of vegetation dynamics. Science, Washington, v. 293, n. 5530, p. 650-655, 2001. 
RENNÓ, C. D.; NOBRE, A. D.; CUARTAS, L. A.; MARTIN, V. S.; TOMASELLA, J.; WATERLOO, M. J. HAND, a new terrain descriptor using SRTM-DEM: Mapping terra-firme rain forest environments in Amazonia. Remote Sensing of Environment, New York, v. 112, n. 9, p. 3469-3481, 2008.

RYAN, T. P. Estatística moderna para engenharia. Rio de Janeiro: Elsevier, 2011. 324 p.

STATGRAPHICS. Statgraphics Centurion XV: user'sguide. New York: Statgraphics, 2006. 299 p.

SOBERÓN, J. Grinnellian and Eltonian niches and geographic distributions of species. Ecology letters, Oxford, v. 10, n. 12, p. 1115-1123, 2007.

SOBERÓN, J.; PETERSON, A.T. Interpretation of models of fundamental ecological niches and species' distributional areas. Biodiversity Informatics, Lawrence, v. 2, p. 1-10, 2005.

SCOLFORO, J. R. S. Biometria florestal: Modelos de crescimento e produção florestal. UFLA/FAEPE, Lavras, 393 p. 2006.

SCOLFORO, J. R. S.; THIERSCH, C. R. Biometria florestal: medição, volumetria e gravimetria. UFLA/ FAEPE, Lavras, 2004. 285 p.

SHCHEGLOVITOVA, M.; ANDERSON, R.P. Estimating optimal complexity for ecological niche models: A jackknife approach for species with small sample sizes. Ecological Modelling, Amsterdam,v. 269, p. 9-17, 2013.

TER STEEGE, H.; PITMAN, N. C. A.; PHILlIPS, O. L.; CHAVE, J.; SABATIER, D.; DUQUE, A.; MOLINO, J. F.; PRÉVOST, M. F.; SPICHIGER, R.; CASTELLANOS, H; HINDEBRAND, P.; VÁSQUEZ, R. Continental-scale patterns of canopy tree composition and function across Amazonia. Nature, London, v. 443, n. 7110, p. 444, 2006.

TUOMISTO, H.; POULSEN, A. D.; RUOKOLAINEN, K.; MORAN, R. C.; QUINTANA, C.; CELI, J.; CAÑAS, G. Linking floristic patterns with soil heterogeneity and satellite imagery in Ecuadorian Amazonia. Ecological Applications, Washington, v. 13, n. 2, p. 352-371, 2003.

VALENCIA, R.; FOSTER, R. B.; CONDIT, R.; SVENNING, J. C.; HÉRNANDEZ, C.; ROMOLEROUX, K.; LOSOS, E.; MAGARD, E.; BASLEVE, H. Tree species distributions and local habitat variation in the Amazon: large forest plot in eastern Ecuador. Journal of Ecology, Oxford, v. 92, n. 2, p. 214-229, 2004.

Recebido em: 06/03/2018

Aceito em: 14/02/2019 